\title{
Accidentes de riesgo biológico entre estudiantes de carreras de la salud. Cinco años de experiencia
}

\author{
Alberto Fica C., M. Irene Jemenao P., Gloria Ruiz R., Milton Larrondo L., \\ Carmen Hurtado H., Gabriela Muñoz G. y Cecilia Sepulveda C.
}

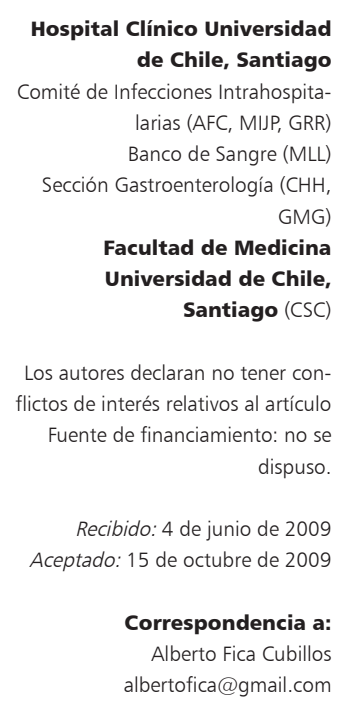

\section{Biological risk accidents among undergraduate healthcare students. Five years experience}

Undergraduate healthcare students are exposed to bloodborne pathogens, and data from developing countries is scarce. We report the experience of a comprehensive program dedicated to the management of this risk. The program includes financial coverage, a 24-hour attention system, HIV, HBV, HCV testing, and free provision of post-exposure antiretroviral drugs. During 2003-2007, incidence rates of these exposures reached 0.9 per 100 student-years. Events were only observed among medicine, nursing, and midwifery students, with rates highest among nursing students (RR 3.5 IC95 $1.93-6.51)$. Cuts and needle stick injuries predominated (74.7\% of accidents). Three students were exposed to HIV patients (1.9\%), all of them received prophylactic drugs, infection was discarded after follow up, and also discarded after exposures to HBV or HCV ( $0.6 \%$ of all accidents). Cost per 1000 student-year was less than 2000 USD. Healthcare students are exposed to biological risks during their studies and a comprehensive program is feasible in a developing country.

Key words: Education Medical Undergraduate, Blood-borne pathogens, Occupational exposure, Needlestick injuries, Disease transmission, Nursing, Midwifery.

Palabras clave: Carreras de la Salud, Agentes de transmisión sanguínea, exposición ocupacional, accidentes corto-punzantes, enfermería, obstetricia.

\section{Introducción}

$\mathrm{U}$ n estudiante de una carrera de la salud, al igual que el personal de salud, está expuesto al riesgo biológico de adquirir agentes de transmisión sanguínea durante sus prácticas ${ }^{1,2}$. Este problema, delineado y prácticamente asegurado en países desarrollados, podría adolecer de serias dificultades en países menos desarrollados para otorgar las medidas preventivas adecuadas o un cuidado apropiado para evitar el contagio con los virus potencialmente involucrados después de que ocurre una exposición. Este trabajo reporta la experiencia acumulada con un programa para el manejo de accidentes de riesgo biológico con agentes de transmisión sanguínea en estudiantes de la salud en la Universidad de Chile.

\section{Métodos}

Programa de prevención y manejo de exposiciones de riesgo biológico a agentes de transmisión sanguínea. Este programa se inició a mediados del año 2002 en la Facultad de Medicina de la Universidad de Chile. Fue posible por la adopción de una política institucional oficial sobre el tema y complementó el programa universal de inmunización para hepatitis B al ingresar a la universidad, que se aplica desde 1998. El programa contempla un sistema de atención gratuita disponible 24 horas al día en un lugar de referencia, con cobertura sobre todos los estudiantes de la Facultad.

Definición de exposiciones de riesgo biológico. $\mathrm{Se}$ utilizó la definición del CDC, Atlanta, E.U.A., y como tal, las exposiciones fueron separadas en percutáneas, exposición de mucosas y por contacto ${ }^{3}$.

Protocolo. El protocolo está basado en la consulta del estudiante en la Unidad de Emergencia del Hospital Clínico de la Universidad de Chile (HCUCH), adosado a la Facultad de Medicina, quien debe traer una muestra de sangre de la fuente del accidente, si ésta es identificable, con el correspondiente consentimiento informado. Posteriormente, el alumno es encuestado con un formulario estándar para reunir información sobre la fuente del accidente, el fluido involucrado y la historia personal del estudiante sobre inmunización contra hepatitis B. Prosigue la evaluación con un estudio serológico para infección por el virus de inmunodeficiencia humana (VIH), virus 
de hepatitis B y C (VHB, VHC) en la muestra de la fuente del accidente (si está disponible). En el caso de VIH se realiza una prueba rápida (de menos de dos horas de desarrollo) con el correspondiente egreso del alumno si la prueba es negativa.

En caso de una prueba rápida positiva para VIH en la muestra de la fuente, se entregan dos o tres antiretrovirales como profilaxis post-exposición, según la gravedad del accidente. La consulta incluye también una muestra de sangre basal del alumno afectado.

La serología para VHB y VHC de la muestra de la fuente se desarrolla en el primer día hábil disponible. En caso de ocurrir una exposición a una fuente positiva para el antígeno de superficie de hepatitis B (HBsAg) se procede también a la medición de títulos anti antígeno de superficie (anti-HBs) en la muestra basal de un alumno vacunado. Aquellos estudiantes con títulos $\geq 10 \mathrm{mUI} / \mathrm{mL}$ se consideraron protegidos contra VHB y no se les efectuó mayor seguimiento.

La detección de anticuerpos para VIH tipos 1,2 y 0 fue realizada por un inmunoensayo cromatográfico rápido de tercera generación (ACON Laboratories, San Diego, USA). La detección de anticuerpos para VHC y de HBsAg fue ejecutada por técnicas inmunométricas usando el sistema Vitros Immunodiagnostic ${ }^{\mathrm{TM}}$ de OrthoClinical Diagnostics (New Jersey, USA). La medición de títulos anti-HBs fue efectuada por un inmunoensayo con micropartículas (IMx, Abbott Laboratories, USA).

Para evitar gastos innecesarios, los estudios serológicos en la muestra basal del estudiante fueron desarrollados sólo si la muestra de la fuente resultó positiva para VIH, VHC o VHB. Esta política se fundamenta en que el programa no está destinado a conocer el estado serológico de los estudiantes y además permite reducir los costos.

El seguimiento serológico de los estudiantes afectados con exposiciones a pacientes VIH fue programado hasta a los dos a tres meses después del accidente y no posteriormente, ya que se incorporó una prueba de tercera generación.

Para aquellos casos con exposición a una fuente de VHC, el seguimiento fue programado a los dos y luego a los seis meses. En casos seleccionados, se efectuó RPC cualitativa para VHC usando un protocolo previamente publicado. ${ }^{4}$ En la Tabla 1 se presenta un resumen del protocolo de manejo.

Tasas y análisis de datos. La información utilizada en este trabajo fue reunida de los registros del Comité de Infecciones Intrahospitalarias del $\mathrm{HCUCH}$, organismo encargado de coordinar y ejecutar este programa. Se utilizó la información del año 2003 al 2007. Los resultados se presentan en una forma descriptiva, incluyendo tasas de incidencia para todas las exposiciones y por agentes específicos. El riesgo relativo fue calculado usando la tasa más baja distinta de cero como referencia. Los intervalos de confianza y la significancia fueron determinados mediante Statcalc del paquete estadístico EpiInfo 6.0.

Análisis económico. Los costos del programa fueron calculados usando los datos de facturación anual hechos a la Facultad de Medicina y separando las visitas de urgencia y seguimiento, las pruebas de laboratorio y los anti-retrovirales profilácticos. Los costos son expresados en US\$ usando valores del año 2007 con una paridad cambiaria de 529 pesos chilenos por dólar.

\section{Resultados}

Tasa de accidentes con exposición a agentes de transmisión sanguínea, riesgo relativo y carreras involucradas. Durante el período 2003 al 2007, se registraron 155 accidentes de este tipo entre los estudiantes de la Facultad. La tasa anual alcanzó un valor de 0,9 por 100 estudiantes (Tabla 2). Las exposiciones sólo fueron observadas en estudiantes de tres carreras: Medicina, Enfermería y Obstetricia. En cinco años no se registraron accidentes entre alumnos de Tecnología Médica, Kinesiología, Nutrición y Dietética, Fonoaudiología o Terapia Ocupacional. Para cada año, la tasa de incidencia fue más elevada entre alumnos de enfermería que entre aquellos de medicina u obstetricia (tasa de incidencia de 2,3 versus 1,2 y 0,06 por 100 estudiantes-año, respectivamente, Tabla 2). El riesgo relativo para accidentes biológicos con exposición a agentes de transmisión sanguínea fue significativamente mayor entre alumnos de enfermería respecto a alumnos

Tabla 1. Programa de manejo de accidentes de riesgo biológico para agentes de transmisión sanguínea para alumnos de pregrado de la Facultad de Medicina de la Universidad de Chile

Atención médica en un sitio centralizado durante 24 horas y con acceso gratuito

Estudio serológico universal de cada fuente identificable para VHB, VHC y con una prueba rápida de tercera generación para $\mathrm{VIH}$.

Protocolos escritos para el manejo de las exposiciones específicas por VHB, VHC y VIH, dotación de anti-retrovirales profilácticos para manejo post-exposición con fuente de $\mathrm{VIH}$ y apoyo técnico.

Manejo clínico y seguimiento serológico

Exposición a VIH: dos o tres anti-retrovirales profilácticos durante un mes y seguimiento a los dos meses.

Exposición VHC: seguimiento serológico a los dos y seis meses

Exposición VHB: títulos anti-HBs en caso de inmunización previa, inmunoglobulina hiper-inmune para VHB en aquellos no vacunados o con títulos inadecuados

Educación sobre precauciones estándares en diferentes niveles del pregrado.

Retroalimentación periódica a las autoridades

VHB: Virus de hepatitis B; VHC: Virus de hepatitis C; VIH: virus de inmunodeficiencia humana; Anti-HBs: anticuerpos anti antígeno de superficie del virus de hepatitis $B$. 
Tabla 2. Caracterización de accidentes de riesgo biológico para agentes de transmisión sanguínea entre estudiantes de pregrado de la Facultad de Medicina de la Universidad de Chile, 2003-2007

\begin{tabular}{|c|c|}
\hline Componente & Total o \% \\
\hline Exposiciones ( $n$ ) & 155 \\
\hline Fuente identificable (n) & 152 \\
\hline$\%$ con fuente conocida & $97,9 \%$ \\
\hline Fuentes con estudio realizado ( $n$ ) & 151 \\
\hline$\%$ de fuentes estudiadas & $99,4 \%$ \\
\hline Población de estudiantes ( $n$ ) & 17.204 \\
\hline Tasa de incidencia por 100 estudiantes-año & 0,9 \\
\hline \multicolumn{2}{|l|}{ Tasa por carrera por 100 estudiantes-año } \\
\hline Medicina & 1,2 \\
\hline Enfermería & 2,3 \\
\hline Obstetricia & 0,6 \\
\hline \multicolumn{2}{|c|}{ Exposiciones a (n) (\% del total, tasa por 100 alumnos-año) } \\
\hline VIH & $3(2,0 \%, 0,02)$ \\
\hline VHB & $1(0,6 \%, 0,01)$ \\
\hline $\mathrm{VHC}$ & $1(0,6 \%, 0,01)$ \\
\hline \multicolumn{2}{|l|}{ Contagio, \% } \\
\hline $\mathrm{VIH}, \mathrm{VHB} \circ \mathrm{VHC}$ & $0,0 \%$ \\
\hline
\end{tabular}

de medicina u obstetricia (RR 3,5; IC95 1,93 a 6,55 para enfermería y 1,9; IC95 1,09 a 3,46 para estudiantes de medicina, $\mathrm{p}<0,0001 \mathrm{y}<0,05$, respectivamente, tomando como referencia la tasa observada entre alumnos de obstetricia)

Tipo de exposiciones a riesgo biológico. La mayor parte de las exposiciones fueron percutáneas e involucraron instrumental o material cortante $(74,7 \%)$. Las salpicaduras sobre mucosas alcanzaron un cuarto de los eventos (24\%). Las exposiciones por contacto en piel no indemne fueron muy infrecuentes $(1,3 \%)$.

Estudio de la fuente, exposición a agentes de transmisión sanguínea y seguimiento. Aproximadamente $98 \%$ de los accidentes tuvo una fuente identificable (152 de 155, Tabla 2). De estos 152 casos, se pudo contar con estudio serológico en todos ellos, salvo en uno $(99,4 \%)$. La exposición a agentes de transmisión sanguínea fue infrecuente entre estos accidentes. La exposición a una fuente con infección por VIH ocurrió en sólo $2 \%(\mathrm{n}=3)$ de todos los accidentes y a VHB y VHC en sólo $0,6 \%$ (uno cada uno, respectivamente, Tabla 2). Los accidentes sin exposición a estos patógenos representaron $96,8 \%$ del total. La tasa de exposición a cualquiera de estos patógenos de transmisión sanguínea por 100 estudiantes-año fue baja $(0,02$ para VIH, y 0,01 para VHB y VHC, respectivamente, Tabla 2). En ningún estudiante expuesto ocurrió algún contagio. La profilaxis post-exposición comenzó antes de dos horas pero la adherencia fue sub-óptima en dos de los tres alumnos expuestos a VIH, debido a la interrupción de indinavir y efavirenz, respectivamente, en la primera semana. Sin embargo, la profilaxis continuó con dos anti-retrovirales en estos alumnos. La exposición a un portador crónico de HBsAg sucedió en un alumno con títulos adecuados de anti HBs y no se efectuó mayor seguimiento. En la única exposición a una fuente positiva para $\mathrm{VHC}$, el seguimiento del estudiante descartó seroconversión.

Situación inmunitaria para VHB. De los 155 estudiantes afectados, 152 estaban vacunados contra VHB $(98,1 \%)$.

Casos especiales. Se observaron algunos resultados indeterminados o límites que generaron dificultades para resolver las exposiciones. Estos casos no fueron considerados como exposiciones confirmadas. Dos de ellos involucraron serologías para VIH. En uno de ellos, una estudiante tuvo una exposición accidental percutánea con un recién nacido $(\mathrm{RN})$ hijo de una madre con infección por VIH conocida. La prueba rápida para VIH fue positiva en una muestra de la fuente (el RN) por la transferencia pasiva de anticuerpos desde la madre. Para descartar la infección en el neonato se requirió una prueba de RPC que finalmente descartó la infección en el RN. En otro caso, una prueba indeterminada por la prueba rápida para VIH requirió una segunda prueba serológica convencional que descartó la infección en la fuente.

También hubo confusión en dos casos asociados a pruebas serológicas positivas para VHC. En la primera, una reacción positiva débil obligó a efectuar una RPC para este agente que confirmó su condición negativa. En el otro caso, un paciente también presentó un resultado indeterminado y no fue posible tomar una segunda muestra para RPC. El seguimiento serológico del alumno, descartó la potencial transmisión. Como se mencionó, ninguno de estos cuatro casos fue incluido como exposición confirmada.

Análisis económico. El costo anual del programa alcanzó los US\$ 4604 ( 2.500.000 de pesos chilenos), correspondiendo el $71,9 \%$ al gasto involucrado en la evaluación y manejo inicial (Tabla 3). Los anti-retrovirales indicados en forma profiláctica representaron menos de $10 \%$ de las necesidades financieras (Tabla 3). El costo anual por estudiante de medicina, enfermería u obstetricia fue menor a US\$ 2 ( $\sim 1.000$ pesos chilenos) y menos de 


\begin{tabular}{|c|c|c|c|}
\hline Componente & Pesos chilenos & $\begin{array}{c}\text { US\$ } \\
(1 \text { US } \$=529 \text { pesos chilenos })\end{array}$ & $\%$ \\
\hline \multicolumn{4}{|l|}{ Evaluación inicial } \\
\hline Consulta en unidad de emergencia & 3.348 .041 & 6.329 & 27,5 \\
\hline Pruebas de laboratorio & 4.534 .059 & 8.571 & 37,2 \\
\hline Anti-retrovirales & 871.263 & 1.647 & 7,2 \\
\hline Subtotal manejo inicial & 8.753 .363 & 16.547 & 71,9 \\
\hline \multicolumn{4}{|l|}{ Seguimiento } \\
\hline Visitas de seguimiento & 3.359 .679 & 6.351 & 27,6 \\
\hline Pruebas de laboratorio & 65.596 & 124 & 0,5 \\
\hline Subtotal seguimiento & 3.425 .275 & 6.475 & 28,1 \\
\hline Total & 12.178 .638 & 23.022 & 100,0 \\
\hline Costo total por año & 2.435 .516 & 4.604 & \\
\hline Costo total por estudiante-año (medicina, enfermería y obstetricia) & 1.042 & 1,97 & \\
\hline Costo por 1000 estudiantes-año de medicina, enfermería y obstetricia & 1.043 .188 & 1.972 & \\
\hline
\end{tabular}

US\$ $2000(\sim 1.000 .000$ de pesos chilenos $)$ por cada 1.000 alumnos de estas carreras (Tabla 3).

\section{Discusión}

Este trabajo demuestra que un programa integral de manejo para accidentes de riesgo biológico a agentes de transmisión sanguínea es posible en un país en vías de desarrollo. Sin embargo, para alcanzar esta condición fue necesaria la adopción de una política institucional y un financiamiento central. Los recursos humanos y la tecnología estaban disponibles, pero no integradas hasta que este programa se desarrolló. Menos de US\$ 4000 anuales fueron requeridos para iniciar y mantener éste programa y menos de US\$2000 por 1.000 alumnos. Estos números parecen posibles de lograr al menos para algunas escuelas en países en desarrollo.

Chile tiene una baja prevalencia de portadores crónicos de $\mathrm{VHB}$ o de personas con infección por $\mathrm{VHC}$ o $\mathrm{VIH}^{4-6}$. Por ejemplo, la prevalencia de HBsAg en donantes de sangre ha sido menor a $0,05 \%$ desde los 90 y la tasa de portadores crónicos de $\mathrm{VHB}<0,5 \%$ de la población. ${ }^{5,6}$ La tasa anual de notificación de VHC en Chile fue $\sim 1$ por 100.000 habitantes en la última década con $<05 \%$ de prevalencia en donantes de sangre., ${ }^{4,6}$ Además, la seroprevalencia de VIH en el mismo grupo también fue $\leq 0,5 \%$ entre 1992 y el 20007 . Estos datos contrastan con los asociados a otros países latinoamericanos como Brasil, Argentina o República Dominicana, caracterizados por cifras endémicas de portadores crónicos de $\mathrm{VHB}^{6}$.

Salvo para hospitales de referencia o salas especializa- das, los pacientes ingresados al sistema de salud no son necesariamente diferentes de la población general. Así, cuando la prevalencia de una enfermedad infecciosa es baja, hay un factor epidemiológico protector sobre el alumno o personal de salud ya que el encuentro con una condición de riesgo será infrecuente. En línea con este fenómeno, $>95 \%$ de los accidentes no representaron una exposición de riesgo biológico para nuestros alumnos (Tabla 1). En contraste, las tasas de exposición para VIH o VHC en el personal de salud en Brasil alcanza $\sim 10 \%$ o más ${ }^{8}$.

Tal como se describe en otros reportes, la exposición percutánea fue la forma predominante involucrando pinchazos de aguja y diversos cortes con material cortante ${ }^{9-11}$. El porcentaje relativamente alto de salpicaduras sobre mucosas, mantenido sobre $18 \%$ durante 5 años, indica una dificultad, ya sea por falta de capacitación, hábito o indumentaria, para incorporar barreras de protección durante las prácticas clínicas.

En esta experiencia se pudieron lograr altos porcentajes de estudio serológico para VIH, VHB y VHC en las fuentes conocidas de un accidente y también altos porcentajes de seguimiento completo. Se invirtió una gran cantidad de tiempo para rastrear a los alumnos después de la exposición, mediante llamadas telefónicas y ocasionalmente por cartas de advertencia. Creemos que esta estrategia fue importante para alcanzar este grado de cumplimiento. Otras experiencias han reportado pérdidas importantes en el número de personas bajo seguimiento ${ }^{8}$.

Sólo un estudiante tuvo exposición a un portador crónico de VHB en esta serie y sólo se requirieron US\$ 23 para evaluar los títulos de anticuerpos anti HBs. La alta cobertura de inmunización para hepatitis B es un 
importante factor en estos programas porque evita el uso de la costosa inmunoglobulina hiper-inmune para VHB en aquellos no vacunados. La vacunación universal para VHB se inició en Chile el 2005 y, si bien los alumnos de las carreras de la salud no fueron protegidos durante su infancia, sí recibieron un programa especial de inmunización al ingresar a la universidad.

Se observó un riesgo relativo mayor entre los estudiantes de enfermería respecto a otras carreras. Las enfermeras, como grupo profesional han estado asociadas a una de las mayores tasas de eventos en diferentes latitudes entre el personal de salud y se observa, con frecuencia, que cerca de $50 \%$ de todos estos accidentes ocupacionales para agentes de transmisión sanguínea ocurren en este grupo $^{8,9,11}$.

Nuestro protocolo de trabajo está basado en una prueba de tercera generación. ${ }^{12}$ Esta estrategia permite mitigar el estrés o angustia del estudiante involucrado y evita cursos innecesarios de profilaxis después de un accidente con exposición biológica. Al mismo tiempo, las pruebas de tercera generación permiten seguimientos serológicos abreviados porque tienen una ventana seronegativa más $\operatorname{corta}^{13}$. Existen varios proveedores de pruebas rápidas para detectar la infección por VIH pero no todas ellas están dotadas de una alta sensibilidad y especificidad o son fáciles de ejecutar ${ }^{14}$. En nuestro caso se utilizó una prueba con $99,9 \%$ de sensibilidad y $99,8 \%$ de especificidad.

La adherencia a la profilaxis es un tema recurrente y en nuestra serie sólo fue adecuada en uno de los tres alumnos que la requirieron. Las interrupciones en los otros dos casos con profilaxis para VIH fueron observadas durante la primera semana pero al menos dos anti-retrovirales fueron mantenidos por el resto del mes. De acuerdo a la literatura científica, cerca de $65 \%$ del personal de salud logra completar la profilaxis ya sea como un esquema completo o reducido y nuestros datos son compatibles con esos valores ${ }^{15}$.

Los accidentes que involucraron neonatos de madres con infección por VIH y las pruebas indeterminadas para VHC generaron confusión y obligaron a realizar pruebas adicionales para aclarar si ocurrió o no una exposición para agentes de transmisión sanguínea. La RPC para $\mathrm{VIH}$ o VHC no fueron consideradas originalmente en el protocolo pero resultaron ser importantes en el manejo de nuestros pacientes.

Este trabajo tiene varias limitaciones. En primer lugar está basado en los eventos reportados y se desconoce cuántos estudiantes decidieron no buscar atención médica luego de una exposición. En segundo lugar, no considera a los alumnos de odontología porque pertenecen a una Facultad separada. La información sobre este grupo indica una alta frecuencia de exposiciones ocupacionales y subnotificación ${ }^{16}$. En tercer lugar, los cálculos financieros están basados en el escenario analizado y no sabemos cómo podría aumentar el gasto con mayores prevalencias de infección por VHB o VIH o una menor cobertura de vacuna para VHB. Finalmente, no fue posible calcular el riesgo relativo entre las tasas observadas en alumnos respecto al personal de salud porque la información sobre este grupo no estaba disponible. Sólo como comparación, la tasa anual de exposiciones de este tipo en uno de los hospitales donde los alumnos hicieron sus prácticas, varió entre 2,6 y $3,7 \%$, sugiriendo un menor riesgo o una subnotificación entre los estudiantes.

En conclusión, fue posible lograr un manejo racional de exposiciones a agentes de transmisión sanguínea luego de una decisión estratégica y financiera, de la integración del laboratorio y de un diseño protocolar.

\section{Resumen}

Los estudiantes de pregrado de las carreras de la salud están expuestos a riesgos biológicos con agentes de transmisión sanguínea. En este trabajo se reporta la experiencia acumulada con un programa integral para este tipo de accidentes y que incluye atención gratuita las 24 horas, estudio serológico de la fuente para VIH, VHC y VHB, y entrega de anti-retrovirales post-exposición a pacientes infectados por VIH. Desde el año 2003 al 2007 la tasa de incidencia alcanzó una cifra de 0,9 eventos por 100 estudiantes-año. Las exposiciones de riesgo fueron observadas sólo entre estudiantes de medicina, enfermería y obstetricia, siendo la mayor tasa en alumnos de enfermería (RR 3,5 IC95 1,93 a 6,51). Tres alumnos estuvieron expuestos a pacientes con infección por VIH (1,9\% de todos los accidentes), todos ellos recibieron profilaxis, descartándose seroconversión en el seguimiento, al igual que en casos con exposición ante VHB y VHC ( $0,6 \%$ del total de accidentes). El costo del programa fue menor a US\$ 2000 por 1.000 estudiantes-año. Los estudiantes de las carreras de la salud están expuestos a riesgos biológicos durante sus estudios y requieren de un programa de manejo, el que es posible de lograr en un país en desarrollo. 


\section{Referencias}

1.- Henderson D K, Fahey B J, Willy M, Schmitt J M, Carey K, Koziol D L, et al. Risk for occupational transmission of human immunodeficiency virus type 1 (HIV-1) associated with clinical exposures. Ann Intern Med 1990; 113: 740-46.

2.- Sulkowski M S, Ray S C, Thomas D L. Needlestick transmission of hepatitis C. JAMA 2002; 287: 2406-13.

3.- Centers for Disease Control. Update US Public Health Service Guidelines for the Management of Occupational Exposures to HBV, HCV, and HIV and Recommendations for Postexposure Prophylaxis. MMWR Morbid Mortal Wkly Rep 2001; 50: 1-52.

4.- Muñoz G, Velasco M, Thiers V, Hurtado C, Brahm J, Larrondo-Lillo M, et al. Prevalencia y genotipos del virus $\mathrm{C}$ de la hepatitis en donantes de sangre y en pacientes con enfermedad hepática crónica y hepato-carcinoma en población chilena. Rev Méd Chile 1998; 126: 1035-42.

5.- Zunino E. Epidemiología de la hepatitis B en Chile y esquemas de vacunación en Latinoamérica. Rev Chil Infect 2002; 19 : 140-55.
6.- Ibarra H. Cambios en la epidemiología de las hepatitis virales en Chile y consideraciones en estrategias de prevención. Rev Méd Chile 2007; 135: 229-39.

7.- Larrondo M. Infección VIH y transfusión sanguínea. En: Sepúlveda C, Afani A, Eds. SIDA. 3a ed. Santiago, Editorial Mediterráneo. 2000. pp 303-310.

8.- Gutierrez EB, Lopes MH, Yasuda MA. Accidental exposure to biological material in healthcare workers at a university hospital: evaluation and follow-up of 404 cases. Scand J Infect Dis 2005; 37: 295-300.

9.- Kosgeroglu N, Ayrance U, Vardareli E, Dincer S. Occupational exposure to hepatitis infection among Turkish nurses: frequency of needle exposure, sharps injuries and vaccination. Epidemiol Infect 2003; 132: 27-33.

10.- García de Codes IA, de Juanes Pardo JR, Arrazola Martínez M del P, Jaén Herreros F, Sanz Gallardo MI, Lago López E. Accidents involving exposure to HIV-contaminated biological material among workers at a thirdlevel hospital in Madrid (1986-2001). Rev Esp Salud Pública 2004; 78: 41-51.

11.- Hernández Navarrete MJ, Camoins Martí M, Martínez Sánchez EV, Ramos Pérez F, García de Codes IA, Arribas Llorente JL. Occupational exposure to blood and biological material in healthcare workers. EPINETAC project 1996-2000. Med Clin (Barc) 2004; 122: 81-6.

12.- Puro V, Francisci D, Sighinolfi L, et al. Benefits of a rapid HIV test for evaluation of the source patient after occupational exposure of healthcare workers. J Hosp Infect 2004; 57: 179-82.

13.- Busch M P, Lee L L, Satten G A, Henrard D R, Farzadegan H, Nelson K E, et al. Time course of detection of viral and serologic markers preceding HIV-1 seroconversion: implication for screening of blood and tissue donors. Transfusion 1995; 35: 91-7.

14.- Ferreira O C Jr, Ferreira C, Riedel M, Visinoni Widolin M R, Barbosa-Júnior A. Evaluation of rapid tests for anti-HIV detection in Brazil. AIDS 2005; 19 (Suppl 4): S70-S75.

15.- Lee LM, Henderson DK. Tolerability of postexposure antiretroviral prophylaxis for occupational exposures to HIV. Drug Saf 2001; 24 : 587-97.

16.- Machado-Carvalhais H P, Martins T C, Ramos-Jorge M L, Magela-Machado D, Paiva S M, Pordeus I A. Management of occupational bloodborne exposure in a dental teaching environment. J Dent Educ 2007; 71: 1348-55. 